\title{
Medical Marijuana as a Viable Option for Cancer Related Issues: Mini Study
}

\author{
Richard McKinnies, Sandra Collins, Jessica Cataldo, Thomas Shaw, Jane Nichols, \\ Stacey McKinney
}

School of Health Sciences, Southern Illinois University, Carbondale, USA

Email: rmck@siu.edu

How to cite this paper: McKinnies, R., Collins, S., Cataldo, J., Shaw, T., Nichols, J. and McKinney, S. (2020) Medical Marijuana as a Viable Option for Cancer Related Issues: Mini Study. Journal of Cancer Therapy, 11, 199-205.

https://doi.org/10.4236/jct.2020.114017

Received: March 18, 2020

Accepted: April 19, 2020

Published: April 22, 2020

Copyright $\odot 2020$ by author(s) and Scientific Research Publishing Inc. This work is licensed under the Creative Commons Attribution International License (CC BY 4.0).

http://creativecommons.org/licenses/by/4.0/

\begin{abstract}
The purpose of this mini study is to evaluate the perceptions of physicians as it relates to medical marijuana and its use for cancer patients. A survey was delivered to a group of physicians acquired from a nationwide database. The majority of physicians do not utilize medical marijuana in their practice and various reasons were identified, which make physicians feel uncomfortable with this practice. Those currently utilizing medical marijuana in their practice also provided a list of condition they prescribe medical marijuana for as a treatment method. The study helps illustrate the need for more research and data on the use of medical marijuana to determine if medical marijuana is a viable source of treatment so physicians feel comfortable utilizing it in their practice, if and when appropriate.
\end{abstract}

\section{Keywords}

Medical Marijuana, Cancer Treatment, Physician Perspective

\section{Introduction}

Cancer is the abnormal proliferation of cells and has been afflicting the human race for centuries. The first recording of cancer was a breast cancer case from ancient Egypt in $1500 \mathrm{BC}$ [1]. Since this time cancer has become more prevalent and a greater burden for society to deal with. Currently in the United States, cancer is the second leading cause of death, only behind heart disease. It is estimated that one in two men and one in three women will develop cancer at some point in their lifetime [2]. The American Cancer Society also estimates that by January 1, 2016, more than 15.5 million Americans were living who had some history of cancer in their past [3].

When treating cancer there are many options for patients to choose. Three of the main options that have been used are surgery, radiation therapy, and che- 
motherapy. One of the oldest methods is surgery that the surgeon endeavors to remove the entire tumor with clear margins. About $40 \%$ of cancer patients are treated with surgery alone and about a third of them actually achieve cure with this method [2]. The first report of treating cancer with radiation was in 1899 when a basal cell was treated. Today, about $60 \%$ of all patients receive some form of radiation therapy treatment for cancer [4]. A third option for cancer patients is chemotherapy, which was used as early as the 1500's with heavy metals that were very toxic [5]. Other options for cancer treatment can consist of hormonal treatments, immunotherapies, specific targeted therapies (i.e. growth signal inhibitors, apoptosis inducing drugs, and angio-inhibitors) and even nanotechnology to allow more direct and specific drug delivery [1].

One type of drug that is classified as a schedule one drug (the same as heroin and ecstasy) and is considered to have high potential for abuse and no accepted medical use is Cannabis Sativa, better known as Marijuana [6]. Even though it is a class one drug and is considered to have no medical use, many animal trials have been completed and have shown benefits with regard to cancer and cancer treatment. In the 1970s medical marijuana was shown to inhibit or slow the growth of cancer cells and multiple animal experiments have illustrated that medical marijuana has anti-cancer actions [7]. Some of the common issues for cancer patients that medical marijuana has been used for are to help improve nausea and vomiting, anorexia, pain, and even stress [8] [9] [10]. When treating cancer patients in Israel, medical marijuana has been used to relieve symptoms related to disease in advance cases or to reduce side effects of chemotherapy [6].

The purpose of this study is to evaluate the perceptions of physicians on the use of medical marijuana for cancer patients and identify what factors are the main influencers for physicians in prescribing medical marijuana.

\section{Methods}

The paragraph above should be removed as the GA somehow added it to the paper. This pertains to another paper.

Survey Design Empirical data was collected via an email survey of physicians across the United States. This data was part of a larger study on perceptions of medical marijuana as a replacement for opioids. The total survey instrument included 4 questions related to the current opioid epidemic, 10 questions related to medical marijuana as a replacement for opioids, and 12 demographic questions. The questions related to the current opioid epidemic asked respondents to indicate on a 3-point scale (yes, undecided, no) whether they believed there was a current opioid crisis in the United States and if they believed that physicians should take an active role in addressing opioid usage. Respondents were also asked to indicate their level of agreement on a 5-point Likert scale with four opioid solutions frequently cited in the literature: 1) opioid prescribing practices, 2) expanded use and distribution of naloxone, 3) expanded medication-assisted treatment (MAT), and 4) creation of a national prescription database. Lastly, respondents were asked to identify additional solutions they felt would be suc- 
cessful in addressing the opioid epidemic in an open-ended format. Demographic questions asked respondents to indicate the state they primary practiced in, years they have practiced medicine, whether they were board certified, areas of board certification, size of community, size of practice, professional memberships, hours of direct patient care during a typical week, age, gender, race, and ethnicity.

The survey instrument was developed following a thorough literature review and weekly meetings by the research team. Prior to its administration, the survey instrument was tested for clarity, readability, and face validity with a panel of physicians not included in the sampling frame. The survey protocol was approved by the human subjects committee at Southern Illinois University, which ensures ethical clearance of all participants and procedures.

Participants and Procedures: The sampling frame was obtained from a nationwide database company and included a total of 11,939 physicians. The database covered multiple medical specialties including pain management and spread across the United States with all states represented. The survey was sent to physicians via email and was completed using Survey Monkey. The survey was fielded for two weeks. After two weeks, a second survey request was emailed to non-respondents. After accounting for any undeliverable instruments, a total of 11,483 were delivered successfully. The survey yielded 164 responses for a response rate of approximately $1.4 \%$. As respondents did not always answer every question on the survey, the total participant number (n) will vary slightly from subject to subject as indicated in the key findings and discussion section. Recognizing a number of factors may have led to the low response rate, including the possibility of nonresponse bias, researchers acknowledge the results of the survey cannot be generalized to all physicians. However, trends observed provide a means by which to study the topic more extensively.

Data Analysis: Closed-ended survey data were analyzed using SPSS (Version 25, SPSS Inc., USA). Frequency counts and descriptive statistics were calculated for all survey questions. Open-ended responses were analyzed using thematic analysis. Two researchers categorized responses into initial codes independently. The two coding lists were then compared and utilized to condense codes into key themes. Identified themes were validated by the remaining researchers.

\section{Results}

Fifty-five percent of respondents $(\mathrm{n}=152)$ were male, with an average age of 57.3 years $(n=141 ; s=10.9)$. Most respondents were white $(83 \% ; n=148)$ and non-Hispanic $(93.7 \% ; n=142)$. The four regions of the United States were represented fairly equally in the study. Of the 145 physicians who responded on their location, 46 (32\%) were from the Western region, 44 (30\%) were from the Midwest region, 26 (18\%) were from the Southern region, and 26 (18\%) were from the Northeast region. Eighty-nine percent of respondents $(n=148)$ were board certified, with roughly half of those board certified in family medicine ( $\mathrm{n}$ $=91)$. Respondents $(n=137)$ practiced medicine for an average of 26.1 years $(s$ 
$=10.8$ ) and provided an average of 25.7 hours of direct patient care each week ( $\mathrm{n}$ $=161 ; s=17.8)$. Over $58 \%$ of respondents, $(n=145)$ worked in practices with five or fewer physicians.

When physicians were surveyed, $40 \%$ of the respondents said they had recommended medical marijuana to a patient. When physicians were asked which conditions they had recommended medical marijuana for, the following options were selected (see Figure 1); pain (74\%), cancer (65\%), seizure disorder (21\%), HIV/AIDS (19\%), and Alzheimer's disease (8\%). Various other conditions were written in at very small percentages like sleep disorders, chronic pain, nausea and vomiting, anxiety, post-traumatic stress disorder (PTSD), and to help appetite.

Respondents were also questioned about the reason they would not be willing to recommend medical marijuana. The main reason given was due to professional opinion (64\%), organizational policies (45\%), and personal opinion (45\%) (see Figure 2). Other reasons physicians gave for not recommending medical marijuana was other modalities exist, lack of evidence based studies, still an illegal drug in their state, federal law, distance to dispensaries, and still wanting training before recommending.

One survey question inquired about the factors that would influence the physician's decision to recommend medical marijuana. The greatest influencer was the severity of the condition (68\%), followed by prior substance abuse disorder (67\%), access to medical marijuana in the community (54\%), age of patient (44\%), and 37\% selected other (see Figure 3). Other included options influencers like diagnosis, prefer Marinol, need to see more research on benefits and risks, legal issues, Food and Drug Administration (FDA) approval, lack of response to other medications, history of psychosis, and the need for more training. A number of participants responded that they would never prescribe it for their patients.

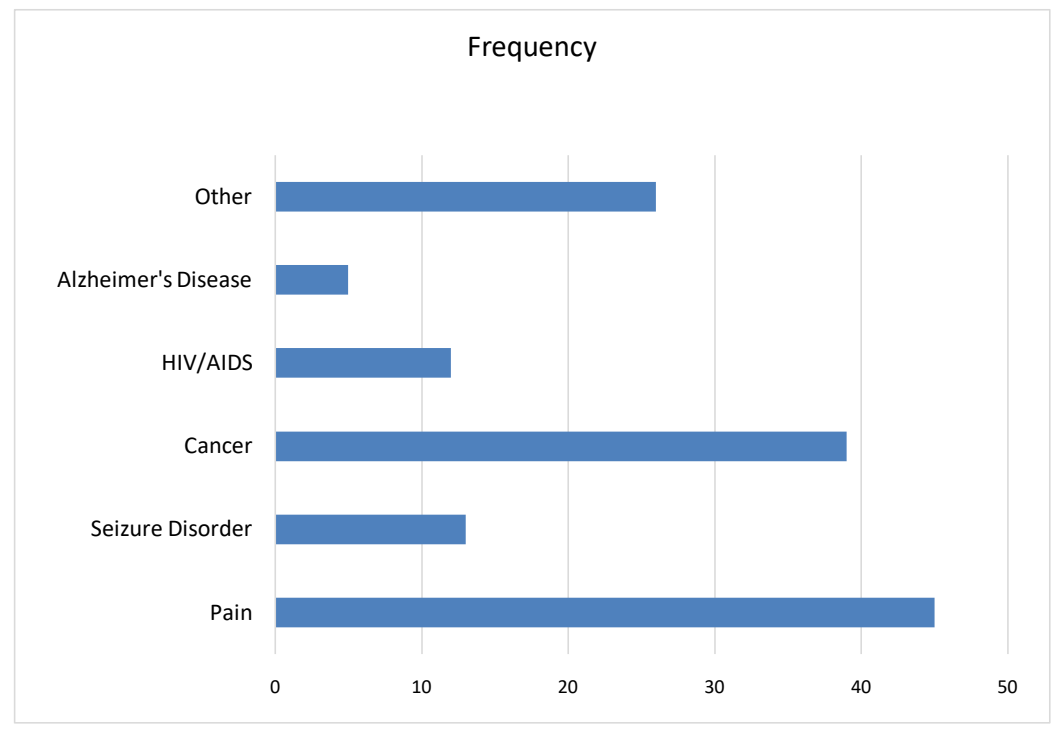

Figure 1. For which medical conditions have you recommended medical marijuana in percent? 


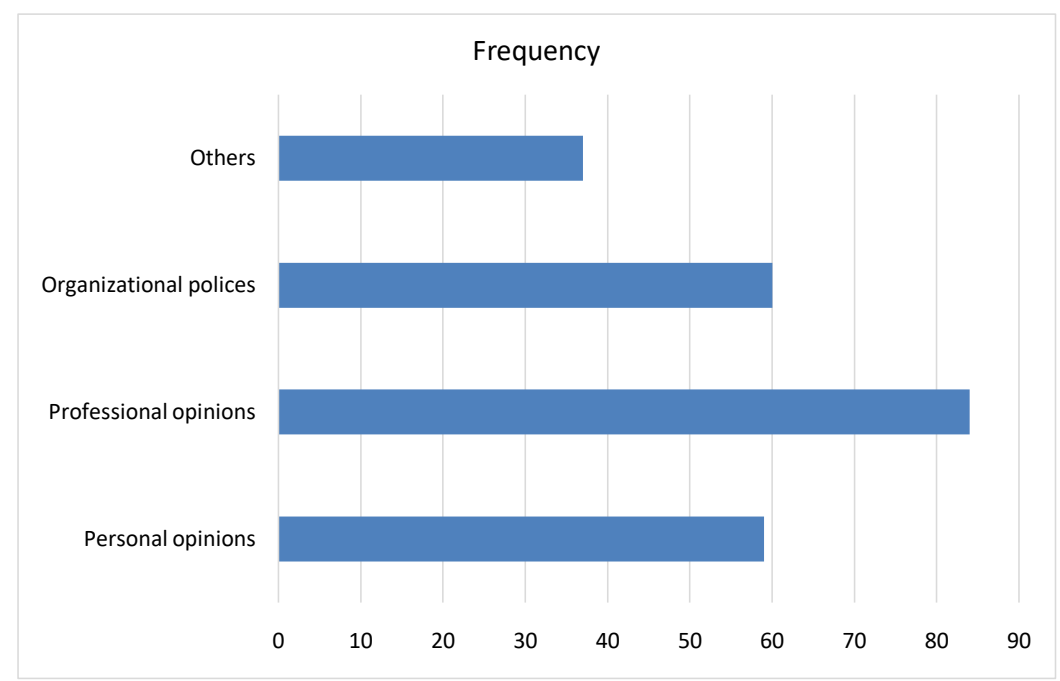

Figure 2. Do personal opinions, professional opinions, or organizational polices influence your decision?

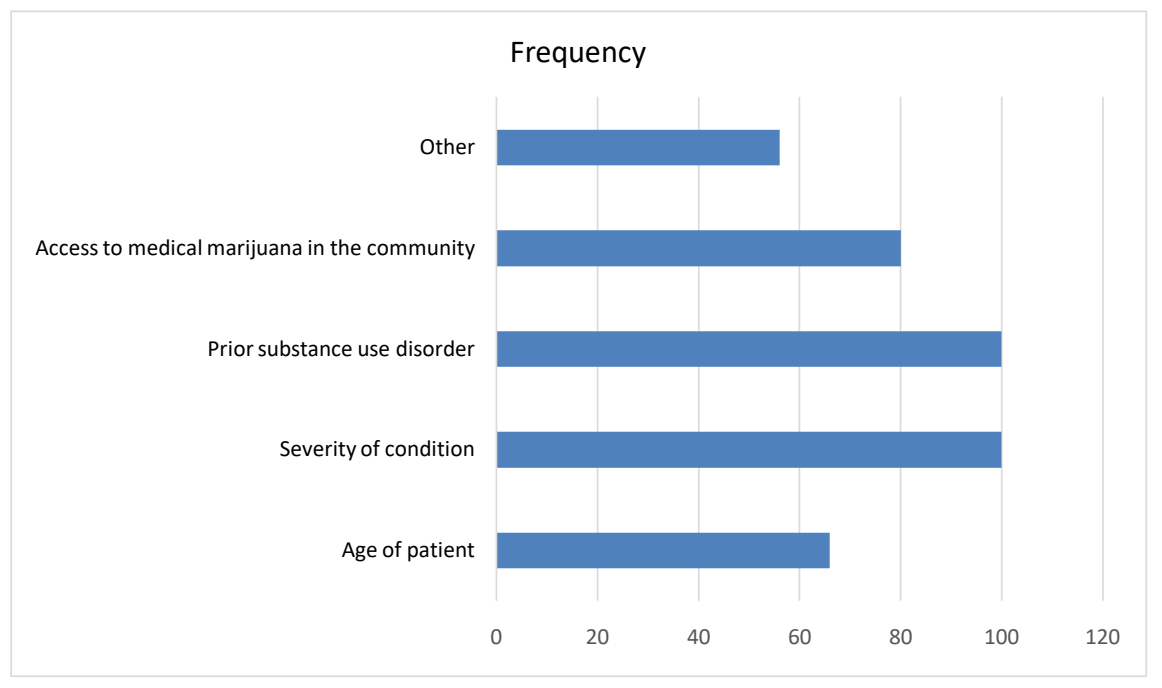

Figure 3. Do personal opinions, professional opinions, or organizational polices influence your decision?

\section{Discussion}

While cancer was the second leading reason in this study that physician would prescribe medical marijuana, the data illustrated that still a majority of physicians would not prescribe it for any condition. The study illustrated that many reasons exist making them feel less than adequate, both personally and professionally, in prescribing medical marijuana. Rubin (2017) found similar findings where respondents included reasons like issues with dosing of the drug or being able to verify concentration and active ingredients of prescribed drug. In this study physicians also struggled with what they referred to as knowledge gap. More specifically their concerns dealt with the fact they had not received any schooling on this topic while in medical school, nothing provided by the FDA, which illuminates any reference of randomized controlled trials. In addition, no 
information is offered in the Physician's Desk Reference, which would provide information pertaining to adequate dosing, indication for use, and any contraindication to using medical marijuana. The fact it is still classified as a schedule one drug, which also limits the possibility of any studies from being done [11]. With such a strong correlation between the research, it seems fairly obvious that more information is needed for physicians to make educated decisions regarding the use of marijuana for medical purposes.

Again, $60 \%$ of participants in this research indicated they would not be willing to prescribe medical marijuana for any of their patients and their concerns seem to have been substantiated by recent research. A study completed by Volkow, Baler, Compton, and Weiss (2014), found that recreational use of marijuana is dangerous for developing brains in young people as well as for those with substance abuse issues or mental illness. They also found chronic use could reduce ones IQ, and also cause someone to develop physical dependence or addictions to the drug [12]. It does however seem to be somewhat of a leap for someone to think recreational marijuana use would automatically translate to similar conclusions in a medical clinic. It also needs to be explained that these toxicities are not associated at the same level for pharmaceutical cannabinoids [13].

\section{Conclusions}

Medical marijuana has been discussed over the years to be a feasible option for cancer patients in treatment or for side effects of both disease and treatment. As demonstrated by this study, for physicians to be comfortable with prescribing medical marijuana to their patients, the classification of the drug is going to have to experience change so more studies can be completed and more information can be provided as a reference. At the current level, most physicians feel inadequate in prescribing medical marijuana as a substitute for other medications or even in general.

This study does have the limitation of a low response rate. This in part maybe due to the email addresses being outdated or due to individual email filters. Even with this limitation, the researchers feel the data can provide some direction on why physicians are not prescribing medical marijuana to their patients. The research can also offer information on specific conditions that physicians deem medical marijuana is appropriate.

Future research could look more specifically at regional issues with prescribing medical marijuana or more state-by state issues that prevent physician recommendation of this drug. Overall, medical marijuana has shown some promise for cancer patients and should be considered for future research with regard to determining its benefits and contraindications.

\section{Conflicts of Interest}

The authors declare no conflicts of interest regarding the publication of this paper. 


\section{References}

[1] US National Library of Medicine National Institute of Health (2010) History of Cancer, Ancient and Modern Treatment Methods. https://www.ncbi.nlm.nih.gov/pmc/articles/PMC3997531/

[2] Washington, C.M. and Leaver, D.T. (2015) Principles and Practices of Radiation Therapy. 4th Edition, Mosby, St. Louis, MO.

[3] American Cancer Society (2019) Cancer Facts and Figures 2019. https://www.cancer.org/content/dam/cancer-org/research/cancer-facts-and-statistic s/annual-cancer-facts-and-figures/2019/cancer-facts-and-figures-2019.pdf

[4] Newton, S., Hickey, M. and Brant, J. (2017) Oncology Nursing Advisor. 2nd Edition, Mosby, St. Louis, MO.

[5] Gates, R. and Fink, R. (2008) Oncology Nursing Secrets. 3rd Edition, Williams, Lippincott.

[6] Bar-Sela, G., Vorobeichik, M., Drawsheh, S., Omer, A., Goldberg, V. and Muller, E. (2013) The Medical Necessity for Medicinal Cannabis: Prospective, Observational Study Evaluating the Treatment in Cancer Patients on Supportive or Palliative Care. Evidence-Based Complementary and Alternative Medicine, 2013, 1-8. https://doi.org/10.1155/2013/510392

[7] Dorey, E. (2012) Cancer High or Hype. Chemistry \& Industry, 12, 16-17.

[8] Murion, B. (2015) Medical Cannabis. Australian Prescriber, 38, 212-215. https://doi.org/10.18773/austprescr.2015.072

[9] Yargic, I. (2013) Synthetic Cannabinoids: More Dangerous than Marijuana. Bulletin of Clinical Psychopharmacology, 1, 18.

[10] Pergam, S., Woodfield, M., Lee, C., et al. (2015) Cannabis Use among Patients at a Comprehensive Cancer Center in a State with Legalized Medicinal and Recreational Use. Cancer, 123, 4488-4497. https://doi.org/10.1002/cncr.30879

[11] Rubin, R. (2017) Medical Marijuana Is Legal in Most States, But Physicians Have Little Evidence to Guide Them. JAMA, 317, 1611-1613. https://doi.org/10.1001/jama.2017.0813

[12] Volkow, N.D., Baler, R.D., Compton, W.M. and Weiss, S.R.B. (2014) Adverse Health Effects of Marijuana Use. The New England Journal of Medicine, 370, 2219-2227. https://doi.org/10.1056/NEJMra1402309

[13] Strouse, T. (2015) Pot in Palliative Care: What We Need to Know. Journal of Palliative Medicine, 18, 7-10. https://doi.org/10.1089/jpm.2015.0003 